\title{
Comparing use and acceptability of menstrual cups and sanitary pads by schoolgirls in rural Western Kenya
}

\section{Linda Mason ${ }^{1 *}$, Elizabeth Nyothach ${ }^{2}$, Anna Maria van Eijk ${ }^{1}$, David Obor ${ }^{2}$, Kelly T. Alexander ${ }^{1}$, Isaac Ngere ${ }^{3}$, Kayla Laserson ${ }^{4}$, Penelope Phillips-Howard ${ }^{1}$}

\author{
${ }^{1}$ Department of Clinical Sciences, Liverpool School of Tropical Medicine, Liverpool UK \\ ${ }^{2}$ Centre for Global Health Research, Kisian Campus, KEMRI Kenya \\ ${ }^{3}$ District Medical Office, Gem District, Yala, Siaya County, Kenya \\ ${ }^{4}$ Division of Global Health Protection, Center for Global Health, CDC India Country Office, New Delhi, India
}

Received: 15 May 2019

Accepted: 12 June 2019

\author{
*Correspondence: \\ Dr. Linda Mason, \\ E-mail: linda.mason@1stmed.ac.uk
}

Copyright: () the author(s), publisher and licensee Medip Academy. This is an open-access article distributed under the terms of the Creative Commons Attribution Non-Commercial License, which permits unrestricted non-commercial use, distribution, and reproduction in any medium, provided the original work is properly cited.

\begin{abstract}
Background: Girls in low and middle-income countries (LMIC) lack access to hygienic and affordable menstrual products. We explore Kenyan schoolgirls' use and views of the cup compared to girls provided with disposable sanitary pads for a feasibility study.

Methods: Schoolgirls aged 14-16 years, received a menstrual cup in 10 schools or 16 pads/month in another10 schools. All were trained by nurses on puberty, hand washing, and product use. They self-completed a net book survey at baseline and twice a term during a year follow-up. We examined their reported ease of insertion and removal, also comfort, soreness, and pain with product use. An aggregate 'acceptability' score was compiled for each product and girls' socio-demographic and menstrual characteristics were compared.

Results: 195 participants received cups and 255 pads. Mean age was 14.6 years, menarchial age was 13.6 years, with an average 3.8 days menses per month. Cup use was $39 \%$ in month 1, rising to $80 \%$ by month 12 (linear trend $\mathrm{p}<0.001$ ). Pad use rose from $85 \%$ to $92 \%$ (linear trend $\mathrm{p}=0.15$ ). Measures of cup acceptability demonstrated girls had initial problems using the cup but reported difficulties with insertion, removal and comfort reduced over time. Girls using pads reported fewer acceptability issues. At baseline, approximately a quarter of girls in the pad arm reported inserting pads intravaginally although this was significantly lower among girls with prior experience of pad use (aRR $0.62 ; 0.45-0.87)$.

Conclusions: While a smaller proportion of girls provided with cups used them in the first months compared to girls given pads, reported use was similar by study-end, and early acceptability issues reduced over time. Girls in LMIC may successfully and comfortably use cups, but require instruction, support and some persistence.
\end{abstract}

Keywords: Africa, Adolescent health, Menstrual cups, Sanitary pads, Schoolgirls, Sexual and reproductive health

\section{INTRODUCTION}

In recent years, research has raised awareness that many adolescent girls in low and middle-income countries (LMIC) suffer physical discomfort and psychological distress because of their monthly menses. ${ }^{1-5}$ Contributing factors include lack of underwear, use of absorbent products or materials which chafe or leak, inadequate facilities to wash and change in privacy and with dignity, cultural mores that restrict daily activity and promote 
seclusion, as well as lack of effective pain management. ${ }^{2,3,6-8}$ Research studies assert these factors compete to reduce school engagement and lower academic achievement; which has implications for girls' future employment, health, and wellbeing. ${ }^{1-4,8,9}$

There is recognition that culturally acceptable hygienic menstrual products need to be accessible for girls and women in LMIC. ${ }^{6,10}$ One of the main pillars of good menstrual hygiene management (MHM) is the provision of adequate affordable hygienic products to absorb or collect menstrual flow. ${ }^{6}$ Two products that have been studied in schoolgirls in LMIC are sanitary pads, and menstrual cups. ${ }^{4,11-17}$ Disposable pads are often assumed as the first choice of product (e.g 'Sanitary pads may not be accessible, forcing some girls to resort to alternative methods of managing menstrual blood'. ${ }^{18}$ However, disposable sanitary pads are not necessarily ideal, as they have been associated with vaginal discharge, leaking, disposal problems, and difficulty (shame and embarrassment) in purchasing. ${ }^{9,13,19-22}$ Key concerns also relate to recurring monthly expense and environmental pollutant effects. ${ }^{9,23}$ A number of countries, including South Africa, Kenya, and India include free or subsidized sanitary pads for schoolgirls as part of their policy to promote MHM, raising concerns about equity in distribution nationally, sustainability, and waste disposal.24-28

The menstrual cup has been evaluated as a possible alternative to sanitary pads or tampons among western women, but it is unclear if findings are transferable to girls in LMIC. ${ }^{29,32}$ Three published studies have explored use in schoolgirls in LMIC; a quasi-experimental study among approximately 100 Nepalese girls evaluated uptake and effect on school attendance over one year, a qualitative study in Uganda evaluated girls' and women's acceptance of cup use, and an RCT evaluated feasibility among Kenyan schoolgirls., ${ }^{4} 15,16,33$ Each of these illustrated that cups could be used among girls, but did not examine issues relating to the problems faced with initial use. A cross-over study among 110 South African women provided menstrual cups compared with their usual items (pads or tampons) reported on comfort, quality, blood collection, appearance and preference; with participants' feedback rating cups more highly by the end of the study. ${ }^{14}$

In Kenya, the current randomized controlled feasibility study in schoolgirls examined water, sanitation and hygiene (WASH) in schools, qualitative feedback on acceptability of cups and pads, safety and potential infections, and inferences on potential impact on sexual and reproductive health and schooling., ${ }^{4,34-37}$ This present study utilizes data from this study, exploring girls' reported use of the cup or pad (depending on study allocation) and their perception of 'acceptability' in terms of insertion, emptying, comfort, pain and soreness when followed-up over time.

\section{METHODS}

\section{Study site and population}

The study was conducted in Gem, Siaya County, a rural area in western Kenya close to Lake Victoria, and 40km from Kisumu, the third largest city in Kenya. The population consists mostly of members of the Luo ethnic group who are mainly subsistence farmers. ${ }^{38,39}$ The study site was within a health and demographic surveillance system, enhancing participant follow-up., ${ }^{43}$ Girls in this area use sanitary pads when available affordable but also rely on traditional methods of menstrual protection.

\section{Menstrual solutions study (MSS)}

This study was nested in a cluster randomized controlled feasibility pilot study, which examined the effect of menstrual products on primary schoolgirls' sexual and reproductive health and schooling outcomes. ${ }^{4}$ Thirty primary schools were randomized into three arms; menstrual cups, sanitary pads, or usual practice. Participants were enrolled into the main study following parental consent and their own assent and were eligible if aged 14-16 years, resident of the study area, had established menses (three or more), and had no disability precluding their participation. Schoolgirls were eligible for this nested sub-study if they were in the cup or pad arms.

\section{Menstrual product implementation}

Girls enrolled in the menstrual cup arm were provided with one menstrual cup (Mooncup®; Mooncup Ltd), size $\mathrm{B}$ for nulliparous girls, or size A for those who had given birth. Girls in the sanitary pad arm were each given 2 packs (total 16 pads) of Always ${ }^{\circledR}$ (Procter and Gamble Ltd) monthly, a brand available in Kenya. Each girl received training according to the study arm. All girls were trained by study nurses on puberty education, and hand washing hygiene. Girls enrolled into the pad arm were shown how to use, change, and dispose of their pads. Those in the cup arm were instructed on how to insert, empty, and reinsert their cup, how to keep it clean and store correctly. 'Champion' girls from a school in south Nyanza, who were experienced menstrual cup users, provided informal peer support, giving practical advice and encouragement on cup use. Rolling recruitment of participants resulted in intervention provision between August 2012 and August 2013, with participants followed until study end, November $2013 .{ }^{4}$

\section{Follow-up of participants to evaluate use of and acceptability of cups and pads}

Study staff trained participants on how to confidentially self-complete an electronic questionnaire on a net book at baseline. Girls were followed up for data collection individually by study nurses approximately twice each term over a calendar year (e.g. three terms), when they 
were requested to complete a survey taking approximately 45 minutes. The survey included questions on their menstruation, hygiene, use of pads/cup (depending on study arm) and aspects of 'acceptability' of the cup or pad during the previous month's use, similar to Howard and colleagues 'satisfaction' score. ${ }^{32}$ Our 'acceptability' score was reduced to five variables - was the cup or pad: easy to insert /put in place; easy to remove, comfortable to wear; causing any soreness and causing any pain. Responses by girls comprised a 3-point scale with a positive (easy, comfortable, no soreness or no pain), just ok, and negative (not easy, uncomfortable, sore, or painful) response.

\section{Data management and analysis}

Data captured through the tablets were uploaded weekly to the KEMRI server, where consistency checks were performed for quality control. Data were tabulated by participants' study ID, school, and study arm. Socioeconomic status (SES) of participants was available from routine household surveys in the health and demographic surveillance site; multiple component analysis aggregated SES related data into five quintiles, which were then dichotomized into the poorest (1-2) and less poor (3-5); those whose SES was not gathered from the household surveys were classed as 'unknown'. The five 'acceptability' variables were aggregated into a composite score. For each positive response (easy, comfortable, not painful etc), a score of 20 was given, for each mid response (' $\mathrm{OK}$ ') a score of 10 was given, and no score was given for a negative response (not easy, painful, sore etc). The sum of scores for every participant per interview was calculated (maximum 100), facilitating calculation of mean values per arm over time, a linearity F score, and significance at the 0.05 level.

\section{Statistical analysis}

The analysis was restricted to girls having more than two school nurse visits. We used all follow-up visits to 12 months cut-off, e.g. one calendar year comprising three terms. Summary statistics and $95 \%$ confidence intervals were generated for relative risks. Continuous variables were expressed as mean and standard deviation (sd), or median and range depending on distribution. Categorical variables were compared by using the Pearson's $\chi 2$ test and trend analysis used $\chi 2$ linear by linear association. Multivariate logistic regression used month 3 data to examine predictors for cup and pad use as an indicator for uptake and ongoing use, and to examine characteristics associated with internal use of pads. The independent variables were included in the model if significant differences in proportions were detected using the Pearson's $\chi^{2}$ test in the bivariate analysis; age, SES, heavy period, cramps, prior pad use, number of pads; and excluded age at menarche, total days menses, school class at enrolment. Relative risks and confidence intervals were reported. Data analyses was performed using SPSS
(Statistical Package for Social Sciences v24.0) and significance was set at $\mathrm{P}<0.05$.

\section{RESULTS}

\section{Socio-demographic and menstrual characteristics at baseline}

Of 751 girls in the main trial allocated to an intervention arm, 725 completed a baseline survey. Among these, 231 allocated to the control arm were excluded from this nested sub-study. Of 494 girls allocated cups or pads, 44 participants who had fewer than three visits over the course of the study were excluded, providing a population of 450 girls; 195 and 255 allocated cups and pads, respectively. Follow-up evaluation was restricted to 1 year. The 195 girls provided with cups attended 1509 follow-up visits by the end of the 12 months follow-up, averaging 6 (range 3-10) visits, and 255 girls provided with pads attended 2186 visits, also averaging 6 (range 310) visits. Girls had a mean age at baseline of 14.6 years (sd 0.7), two thirds of girls were in higher classes (7 and 8 ), and $14 \%$ were grouped within the poorest two socioeconomic quintiles, with no differences by study arm (Table 1). Participants' mean age at menarche was 13.6 years (sd 0.9), and girls menses lasted a mean of 3.8 (sd 1.4) days. Two thirds reported cramps during menses, and a quarter stated their menstruation was heavy (Table 1). Four in five girls claimed they had ever used pads; this was significantly lower among the pad arm compared with those allocated cups $(77 \%$ vs. $88 \% \mathrm{p}=0.002$, Table $1)$. Girls reporting recent sanitary pad use stated they used a mean of 8 pads per menses, averaging 2 per day over 4 days of menstruation. Of a maximum 5 points, girls reported a mean 2.25 (sd 0.6) score for being comfortable with their sanitary hygiene material at baseline with no difference between arms (2.30 vs. 2.19. $\mathrm{p}=0.19$; Table 1).

\section{Use of cups over study follow-up}

Girls' reported uptake and use of cups rose from 39\% after one month, to $80 \%$ by the end of month 12 (Figure $1)$, with a significant linear relationship between use and duration $(\chi 2$ linear trend 68.2; $\mathrm{p}<0.001)$.

When examining cup user characteristics at the end of month 3 in multivariate analysis, in order to assess early usage, $80 \%$ of the poorest socio-economic group reported using cups compared with $68.6 \%$ of less poor (Relative risk [RR] 0.86, 95\% confidence limits 0.83-0.89; Table $2)$. A higher proportion of $15 y$ old girls $(76.4 \%)$ reported cup use compared with older girls (65\%, RR 1.29, 1.221.35). Menstrual health and experience of using pads also appeared to influence reported cup use. In girls experiencing menstrual cramps, $69.8 \%$ reported they used cups compared with $77 \%$ stating they did not have cramps (RR 0.86, 0.80-0.93). Those reporting heavy periods had a slightly higher rate of reported cup use, compared with those not reporting heavy periods $(72.4 \%$ 
vs. 71.6\%; RR 1.12, 1.06-1.19). Although the proportion of reported cup use differed between those who had prior experience of using branded sanitary pads $(72.8 \%)$ and those who had only ever used traditional materials $(61.5 \%)$, this was not significant in multivariate analysis (RR 1.35, 0.74-2.47).

Table 1: Demographic and menstrual characteristics of study population by intervention allocation at baseline.

\begin{tabular}{|c|c|c|c|c|}
\hline \multicolumn{2}{|l|}{ Characteristics } & Pad $(n=255)$ & Cup (n=195) & p-value \\
\hline \multicolumn{5}{|l|}{ Socio-demographic } \\
\hline Age at enrolment & Mean (sd) & $14.51(0.68)$ & $14.64(0.69)$ & 0.06 \\
\hline \multirow[t]{2}{*}{ Class } & 5 or 6 & $80(31.4 \%)$ & $64(32.8 \%)$ & 0.94 \\
\hline & 7 or 8 & $175(68.6 \%)$ & $131(67.2 \%)$ & \\
\hline \multicolumn{5}{|l|}{ Socio-economic status $\S$} \\
\hline & Poorest & $32(12.5 \%)$ & $21(10.8 \%)$ & 0.58 \\
\hline & Less poor & $183(71.8 \%)$ & $142(72.8 \%)$ & \\
\hline & unknown & $40(15.7 \%)$ & $32(16.4 \%)$ & \\
\hline \multicolumn{5}{|l|}{ Menstrual } \\
\hline Age at menarche & Mean (sd) & $13.65(0.81)$ & $13.54(0.96)$ & 0.20 \\
\hline Total days per menses & Mean (sd) & $3.89(1.3)$ & $3.71(1.4)$ & 1.55 \\
\hline Cramps during menses & Yes & $165(64.7 \%)$ & $120(61.5 \%)$ & 0.49 \\
\hline Menses heavy & Yes & $65(25.5 \%)$ & $40(20.5 \%)$ & 0.22 \\
\hline Ever used sanitary pads & Yes & $196(76.9 \%)$ & $172(88.2 \%)$ & 0.002 \\
\hline \multicolumn{2}{|c|}{ Pads per menses (if prior use) } & $\mathrm{n}=187$ & $\mathrm{n}=118$ & \\
\hline
\end{tabular}

Abbreviations: SD: standard deviation; § Measured through multiple component analysis into 5 quintiles; then collapsed into 1-2 (poorest) and 3-5 (less poor); with unknowns included.

Table 2: Multivariate analysis of characteristics associated with cup or pad use at 3 months.

\begin{tabular}{|c|c|c|c|c|c|}
\hline \multirow[t]{2}{*}{ Characteristics } & \multirow[t]{2}{*}{ Values } & \multicolumn{2}{|c|}{ Cup use at month $3(n=138)$} & \multicolumn{2}{|c|}{ Pad use at month $3(n=171)$} \\
\hline & & $\mathrm{N}$ cup use $(\%)$ & RR $(95 \%$ CI), p-value & $\mathrm{N}$ pad use $(\%)$ & RR (95\% CI), p-value \\
\hline All & & $99 / 138(71.7)$ & & $156 / 171(91.2)$ & \\
\hline \multicolumn{6}{|c|}{ Socio-demographic } \\
\hline \multirow[t]{3}{*}{ Age at enrolment } & 14years & $44 / 63(69.8)$ & 1.05 (1.04-1.05), 0.001 & $79 / 87(90.8)$ & $1.32(0.52-3.32), 0.56$ \\
\hline & 15years & $42 / 55(76.4)$ & $1.29(1.22-1.35), 0.001$ & $56 / 62(90.3)$ & $1.37(0.55-3.40), 0.50$ \\
\hline & 16years (ref) & $13 / 20(65.0)$ & 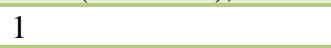 & $21 / 22(95.5)$ & 1 \\
\hline \multirow[t]{3}{*}{ 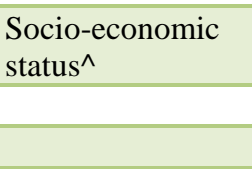 } & Less poor & $70 / 102(68.6)$ & $0.86(0.83-0.89), 0.001$ & $111 / 120(92.5)$ & $1.00(0.82-1.22), 0.99$ \\
\hline & unknown & $17 / 21(81.0)$ & 1.00 (1.00-1.00), 0.10 & $26 / 30(86.7)$ & $1.09(0.92-1.30)$ \\
\hline & Poorest (ref) & $12 / 15(80.0)$ & 1 & $19 / 21(90.5)$ & 1 \\
\hline \multicolumn{6}{|l|}{ Menstrual } \\
\hline \multirow[t]{2}{*}{$\begin{array}{l}\text { Cramps during } \\
\text { Menses }\end{array}$} & Yes & $60 / 86(69.8)$ & 0.86 (0.80-0.93), 0.001 & $104 / 115(90.4)$ & 0.98 (0.92-1.04), 0.46 \\
\hline & No (ref) & $39 / 52(77.0)$ & 1 & $52 / 56(92.9)$ & 1 \\
\hline \multirow[t]{2}{*}{ Menses heavy } & Yes & $21 / 29(72.4)$ & 1.12 (1.06-1.19), 0.001 & $37 / 45(82.2)$ & $0.96(0.83-1.11), 0.58$ \\
\hline & No (ref) & 78/109 (71.6) & 1 & $119 / 126(94.4)$ & 1 \\
\hline \multirow[t]{2}{*}{$\begin{array}{l}\text { Any previous pad } \\
\text { use }\end{array}$} & Yes & $91 / 125(72.8)$ & 1.35 (0.74-2.47), 0.34 & $113 / 124(91.1)$ & $1.01(0.94-1.08), 0.78$ \\
\hline & No (ref) & $8 / 13(61.5)$ & 1 & $43 / 47(91.5)$ & 1 \\
\hline
\end{tabular}

\section{Use of pads over study follow-up}

Uptake and use of pads rose from $85 \%$ after one month to $92 \%$ by month 12 (Figure 1), with no significant linear relationship between use and duration $(\chi 2$ linear trend $2.1 ; \mathrm{p}=0.15)$. By month 3 , reported use of pads was proportionately lower among girls with heavy periods $(82.2 \%)$ compared with lighter menses (94.4\%), but in multivariate analysis this was not significant (RR 0.96, $0.83-1.11)$. No other differences in characteristics were seen (Table 2). 
Girls in the pad arm reported using an increased number of pads over the course of the study from a mean of 2.5 (sd 1.2) in month 1 to 3.5 in month 12 (sd 1.5; linearity: F 40.7, $\mathrm{p}<0.001$ ). A quarter of girls reported wearing their pad (at least some of the time) intravaginally. This was reported by a higher proportion of older girls and those without prior experience of pad use (Table 3). In multivariate analysis, approximately a quarter of girls in the pad arm reported inserting pads intravaginally in their menses prior to study baseline; this was significantly lower among girls with prior experience of pad use (aRR 0.62; 0.45-0.87 Table 3).

Table 3: Multivariate analysis of characteristics associated with intravaginal use of pads.

\begin{tabular}{|llll|}
\hline Characteristics & Values & Reported pad placed intravaginally (n=255) \\
\hline All & & Ninternal $(\%)$ & RR (95\% CI), p-value \\
\hline Socio-demographic & & $61 / 223(27.4)$ & \\
\hline Age at enrolment & 14years & $35 / 125(28.0)$ & $0.75(0.42-1.32), 0.32$ \\
\hline & 15years & $16 / 73(21.9)$ & $0.58(0.32-1.04), 0.07$ \\
\hline & 16years (ref) & $10 / 25(40.0)$ & 1 \\
\hline Socio-economic status ${ }^{\wedge}$ & Less poor & $44 / 161(25.1)$ & $1.38(0.68-2.83), 0.37$ \\
\hline & unknown & $10 / 35(28.6)$ & $1.48(0.48-4.49), 0.49$ \\
\hline & Poorest (ref) & $7 / 27(25.9)$ & 1 \\
\hline Menstrual & & & $1.04(0.72-1.50), 0.85$ \\
\hline Menses cramps & Yes & $42 / 146(28.8)$ & $1.11(0.73-1.70), 0.62$ \\
\hline & No (ref) & $19 / 77(24.7)$ & 1 \\
\hline Menses heavy & Yes & $16 / 52(30.8)$ & $0.62(0.45-0.87), 0.006$ \\
\hline & No (ref) & $45 / 171(26.3)$ & 1 \\
\hline Sanitary pad use ever & Yes & $43 / 176(24.4)$ & $1.22(0.77-1.92), 0.40$ \\
\hline & No (ref) & $18 / 47(38.3)$ & 1 \\
\hline Average $\leq 3$ pads/d used & Yes & $44 / 154(28.6)$ & $17 / 69(24.6)$ \\
\hline
\end{tabular}

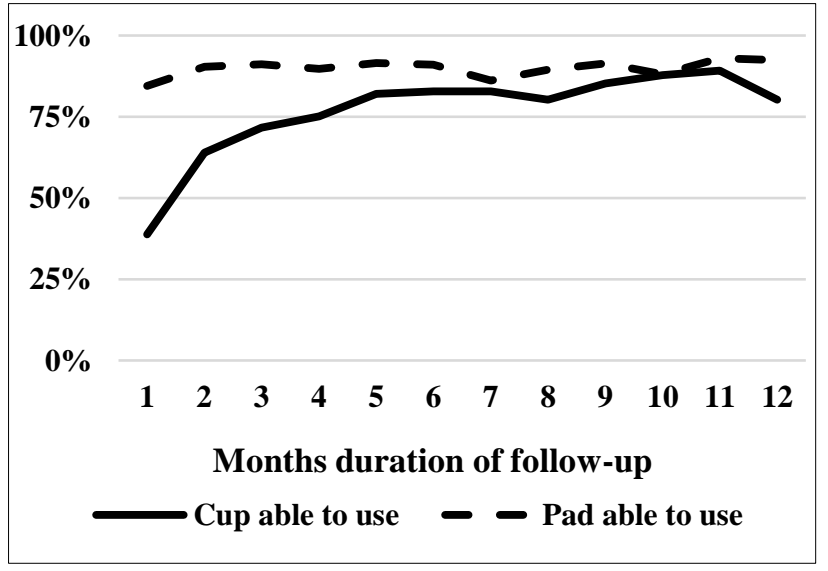

Figure 1: Pad and cup use over 12 months.

\section{Comparison of cup and pad acceptability score over follow-up}

The aggregate mean acceptability score for the five measures rose significantly from 73 to 81 out of $100(\mathrm{~F}$ 17.2; $\mathrm{p}=<0.001$ ) over the study among girls reporting they were able to and used the cup (Figure 2). For girls allocated pads, the aggregate acceptability score in those reporting able to and using pads rose from 83 to 89 out of 100 (F 32.5; p <0.001) over the study (Figure 2).

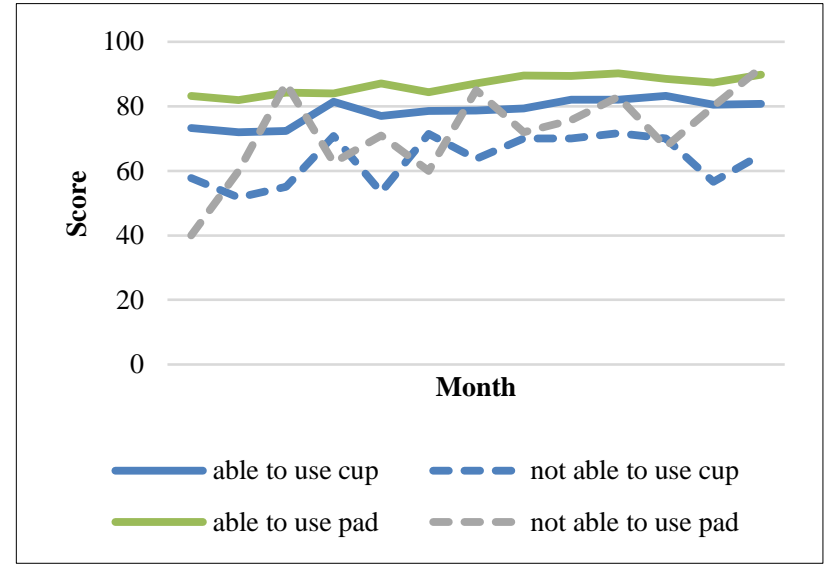

Figure 2: Aggregate score of acceptability measures among girls reporting able and girls reporting not able to use cup or pad.

The five acceptability measures showed that reported problems differed between study arms but reduced over time for both groups. In the cup group, the greatest problems were pain $(23 \%)$ or discomfort $(22 \%)$ during early use i.e. prior to and including month 3 ; however this dropped to $2 \%$ and $7 \%$ respectively by the end of a year (Figure 3). Emptying was problematic for $15 \%$ at 3 months, with between $5-10 \%$ still having some issues 
over the study year. Insertion was slightly less of a problem, $9 \%$ reporting this at 3 months which dropped to $5 \%$ by study end; soreness was rarely reported (Figure 1 2). Among girls trying the cups but unable to successfully use, insertion, removal for emptying, and being uncomfortable were reported by a quarter until month 6; problems with pain were mostly resolved by month 4 .

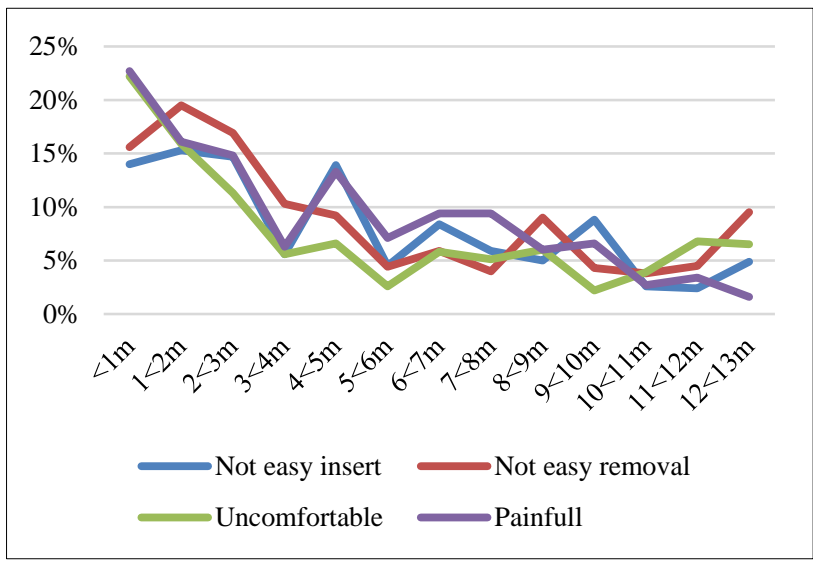

Figure 3: Percentage of girls reporting problems with cups over 12 months.

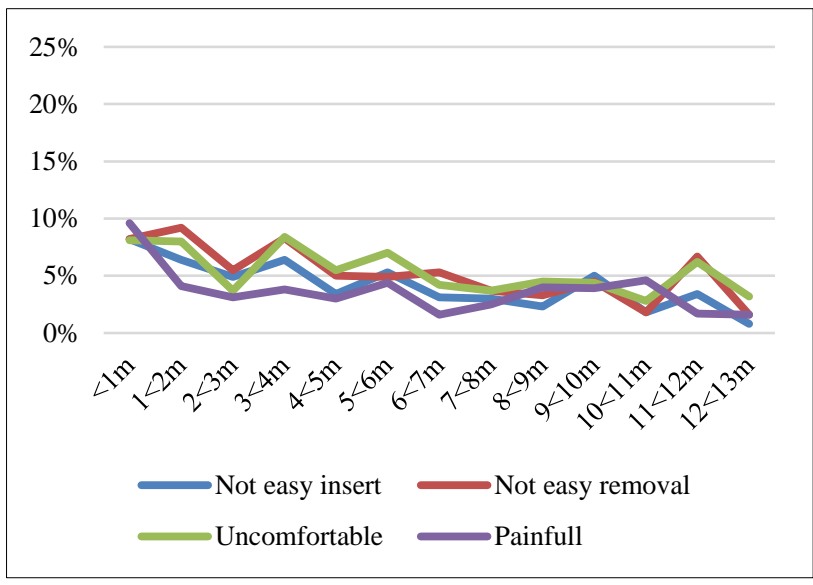

Figure 4: Percentage of girls reporting problems with pads over 12 months.

Few problems were reported by pad users: the greatest problem (noting this was below 10\%) was difficulty removing the pads after use, with between $4-8 \%$ reporting either having problems placing the pad or having pain or discomfort (Figure 4). Among the girls reporting they tried but were not able to use pads in their prior menses, problems with placement, discomfort, and pain were most frequently reported.

\section{DISCUSSION}

To the best of our knowledge, no other study has examined girls' reported experience and problems associated with the use of menstrual cups or sanitary pads internationally. While reported use of pads was consistently high, the proportion of girls reporting cup use increased significantly over time following some initial difficulties. For both products, a proportion of girls reported acceptability issues. Few pad users had problems, but among those who had, there was no reduction in those reporting problems over time. A small proportion of girls continued to report they tried to use their cup or their pad unsuccessfully during the study, due to insertion/placement, emptying, comfort or pain with use problems.

The number of girls who reported using their cup each month increased significantly from $39 \%$ to $80 \%$ over the year. Poorer girls (in the lowest SES quintiles) and those with heavy bleeding were shown to have a higher rate of uptake of cup use by three months in our study, while older girls and those reporting menstrual cramps had a significantly lower uptake at this time. No sociodemographic or physical characteristics have been examined before to understand reasons for slow uptake. Our qualitative findings suggested that girls are apprehensive in using the cup to start with, perceiving it as too big, or fear it causes adverse health effects, but with persistence, often helped by training or peer influence, they were able to master it. ${ }^{15,36}$ Hyttel describes this learning curve as key to success in future programmes. ${ }^{15}$ In Nepal, schoolgirls cup use rose to $60 \%$ by six months and then remained static in the remaining six months; with authors, noting that $91 \%$ of girls continued with the cup once they had initiated use. ${ }^{16}$ The Nepalese study examined the influence of peers demonstrating this was important in successful adoption of the cup by schoolgirls. ${ }^{16}$ A cross-over trial among 110 South African women which included 3 months use of the cup found that 'at least' $93 \%$ wore the cup for their entire menstrual cycle. ${ }^{14}$ The study did not identify any increase or decrease in usage over the 3-month duration of cup use.

While one fifth of girls using the cup recorded discomfort and pain in the first month, this dropped one-tenth by month 3 ; difficulty in inserting or removing their cup varied by month, with the proportion of girls reporting difficulties reducing considerably during the 12 months follow-up. Our findings are comparable with a South African study that showed a dramatic increase in cup uptake: with 38\% reporting 'very easy' insertion: in month 1 to $96 \%$ in month $3 .^{14}$ The same study reported an increase in the proportion of women finding removal very easy from $63-96 \%$ by month 3 . Again, limited qualitative studies appear to confirm issues around insertion and removal in the early stages, and that mastering these techniques is a learning curve, which is greatly assisted by peer support. ${ }^{15,36}$

The composite measure of acceptability showed an increase in 'acceptability' score over time, which followed the trend of all individual attributes. Both the aggregate and individual components were noted to be significantly higher among pad users, compared with cup users, particularly among early adopters. Other studies 
among adult women which compared products in crossover studies found participants reported a preference for cups; in South Africa, over $90 \%$ of women rated the cup better in terms of comfort, quality and overall preference than their usual product (pad or tampon) over 6 menstrual cycles. ${ }^{14}$ Among Canadian cup users $91 \%$ of 47 women reported they would continue to use their cup, while an acceptability rate of $45 \%$ was reported in 51 North American women. ${ }^{31,40}$ In a qualitative examination with participants from our feasibility study, girls provided with cups (most of whom had used pads previously) made their own comparison with wearing a pad, and almost unanimously spoke of preference for the cup after $\sim 6$ months. ${ }^{36}$ The findings in the present study showing difficulties with the five acceptability components had not been addressed in the former published qualitative study. ${ }^{36}$ Instead, participants reported the cup was better able to resolve issues of comfort, leakage, and fear of movement (which might dislodge pads), as well as issues around cost and sustainability. It is possible that girls in the qualitative study may have wished to demonstrate a positive outlook in focus group discussions but were better able to report individual problems on the five acceptability questions when asked in a self-completed questionnaire.

At baseline the average number of pads used per day was 2 , likely due to their prohibitive cost. This would suggest that some girls may wear a pad for up to 12 hours at a time putting them at risk of chafing, discomfort leaking and odour. ${ }^{3,28}$ Laboratory confirmed evaluation of reproductive tract infections found girls provided with pads in our study had a higher prevalence of bacterial vaginosis compared with girls provided with cups. ${ }^{4}$ While our study sought to provide enough pads for each individual girl's needs, they also may be obliged to share, causing extended episodes of wear 'overstaying' and soreness. ${ }^{3,36}$ The number of pads reportedly used per day during their menses increased from 2.5 to 3.5 over the study, suggesting girls may have become more confident in keeping them for their own needs; however, we also note that overuse is also dependent on other factors such as having the facilities, time and opportunity to change. Other research similarly suggests that timely changing is not always possible particularly among schoolgirls where the pupil-latrine ratio may be high or the state of the facilities such that girls choose not to use them. ${ }^{28,35,41}$

Once provided with sanitary pads, nearly all girls reported wearing one for their menses but a small proportion $(<5 \%)$ did not. Few studies have explored why girls with access to disposable pads do not wear them; issues with vaginal discharge, leaking and their disposal have been reported, although it is not known to what extent such issues might prevent those provided with pads from using them. ${ }^{19,42,43}$ Here we found the small proportion reporting issues had difficulty with ease of placement and removing despite initial training. It is quite possible this related to not having suitable, or indeed any underwear, as reported in previous studies. ${ }^{22,44}$ While no question was asked on this topic, we did ask if the pad was worn internally as informal discussions had indicated some girls were doing this. A quarter of girls in the pad group reported they had done this; our analysis suggests girls who had not used pads before the study were the most vulnerable to doing it. This indicates adequate training and consideration of lack of underwear is relevant when providing sanitary pads, particularly as there is an increasing interest by some ministries, including in Kenya, to conduct national pad programmes for adolescent girls. It is also possible that some girls doubled up by wearing internally and placing on their underwear if they were fearful of leaking and wanted reassurance, although this showed no association with reporting 'heavy' menses. This phenomenon might be worth of follow up on, as this may have repercussions for hygiene safety or for the success of any pad programme that does not also provide underwear also.

We note some limitations in our study. First, as with all studies using self-reported data, participating girls may have been prone to exaggerate use. We have evidence that cup colour change due to exposure to vaginal fluids was slower to change over time in comparison to the usage reported by the girls themselves. ${ }^{45}$ This implies that a portion of participants self-reported using the cup, while in practice use may have been minimal. However, as all reports within this paper were self-reported, including use of pads where no change in colour could be examined, we believe the information offers useful comparative insights into perceived use and factors reported to be associated with this. Self-reported use is also the main method cup programmes use to monitor and measure successful implementation. For the acceptability score we allocated an equal weighting to inserting/placement, emptying, comfort, pain and soreness; it is possible that some problems may have been perceived by participants to be of greater concern than others, which we were not able to examine during this study. For logistical reasons girls who were followed up only one or two times were excluded to better enable us to evaluate girls' changing behaviors over time. We also had a 12 months follow-up cut off which reduced duration under study but facilitated analysis by removing outliers and prevented distortion of proportions among small numbers.

\section{CONCLUSION}

To conclude, cup acceptability was high among this population of primary schoolgirls, with few reporting issues around discomfort, pain, difficulties inserting or placement or removing the cup or pad. While the proportion of girls reporting initial issues was higher among girls provided cups compared with pads, these were resolved in the first few months of use. This leads us to conclude that with adequate instruction, support and persistence, girls can successfully and comfortably use either of these products in LMIC. 


\section{ACKNOWLEDGMENTS}

Authors would like to thank to staff, schools, participants and the community for their support of this study, and to ministry officials for their help. Authors thank Mooncup Ltd for providing menstrual cups for participants at a discounted rate. The KEMRI Director approved publication of this paper.

Funding: This study was funded by the UK-Medical Research Council/Department for International Development/Wellcome Trust; grant number G1100677/1. Funders had no role in study design, data collection and analysis, decision to publish, or manuscript preparation

Conflict of interest: None declared

Ethical approval: The study was approved by the Institutional Ethics Committee

\section{REFERENCES}

1. Sommer M. Where the education system and women's bodies collide: The social and health impact of girls' experiences of menstruation and schooling in Tanzania. J Adolesc. 2010;33(4):521-9.

2. McMahon SA, Winch PJ, Caruso BA, Obure AF, Ogutu EA, Ochari IA, et al. 'The girl with her period is the one to hang her head' Reflections on menstrual management among schoolgirls in rural Kenya. BMC Int Health Hum Rights. 2011;11(1):7.

3. Mason L, Nyothach E, Alexander K, Odhiambo FO, Eleveld A, Vulule J, et al. 'We keep it secret so no one should know' - a qualitative study to explore young schoolgirls attitudes and experiences with menstruation in rural Western Kenya. PLoS One. 2013;8(11):e79132.

4. Phillips-Howard P, Nyothach E, ter Kuile F, Omoto J, Wang D, Zeh C, et al. Menstrual cups and sanitary pads to reduce school attrition, and sexually transmitted and reproductive tract infections: a cluster randomised controlled feasibility study in rural western Kenya. BMJ open. 2016;6:e013229.

5. Hennegan J, Dolan C, Wu M, Scott L, Montgomery P. Measuring the prevalence and impact of poor menstrual hygiene management: a quantitative survey of schoolgirls in rural Uganda. BMJ open. 2016;6(12):e012596.

6. Sommer M, Caruso BA, Sahin M, Calderon T, Cavill $\mathrm{S}$, Mahon $\mathrm{T}$, et al. A Time for Global Action: Addressing Girls' Menstrual Hygiene Management Needs in Schools. PLoS Med. 2016;13(2):e1001962.

7. Sommer M. Menarche: a missing indicator in population health from low-income countries. Public health reports. 2013;128(5):399-401.

8. Miiro G, Rutakumwa R, Nakiyingi-Miiro J, Nakuya K, Musoke S, Namakula J, et al. Menstrual health and school absenteeism among adolescent girls in Uganda (MENISCUS): a feasibility study. BMC Womens Health. 2018;18(1):4.
9. van Eijk AM, Sivakami M, Thakkar MB, Bauman A, Laserson KF, Coates S, et al. Menstrual hygiene management among adolescent girls in India: a systematic review and meta-analysis. BMJ Open. 2016;6(3):e010290.

10. Phillips-Howard PA, Caruso B, Torondel B, Zulaika G, Sahin M, Sommer M. Menstrual hygiene management among adolescent schoolgirls in lowand middle-income countries: research priorities. Global health action. 2016;9:33032.

11. Wilson E, Reeve J, Pit A. Education. Period. Developing an acceptable and replicable menstrual hygiene intervention. Development in Practice. 2014;24(1):63-80.

12. Montgomery P, Ryus CR, Dolan CS, Dopson S, Scott LM. Sanitary pad interventions for girls' education in Ghana: a pilot study. PLoS One. 2012;7(10):e48274.

13. Hennegan J, Dolan C, Wu M, Scott L, Montgomery P. Schoolgirls' experience and appraisal of menstrual absorbents in rural Uganda: a cross-sectional evaluation of reusable sanitary pads. Reprod Health. 2016;13(1):143.

14. Beksinska ME, Smit J, Greener R, Todd CS, Lee ML, Maphumulo V, et al. Acceptability and performance of the menstrual cup in South Africa: a randomized crossover trial comparing the menstrual cup to tampons or sanitary pads. J Womens Health (Larchmt). 2015;24(2):151-8.

15. Hyttel M, Thomsen C, Luff B, Storrusten H, Nyakato V, Tellier M. Drivers and challenges to use of menstrual cups among schoolgirls in rural Uganda: a qualitative study. Waterlines. 2017;36(2):109-24.

16. Oster E, Thornton R. Determinants of technology adoption: Peer effects in menstrual cup up-take. Journal of the European Economic Association. 2012;10(6):1263-93.

17. Averbach S, Sahin-Hodoglugil N, Musara P, Chipato T, van der Straten A. Duet for menstrual protection: a feasibility study in Zimbabwe. Contraception. 2009;79(6):463-8.

18. Secor-Turner M, Schmitz K, Benson K. Adolescent Experience of Menstruation in Rural Kenya. Nurs Res. 2016;65(4):301-5.

19. Lawan UM, Yusuf NW, Musa AB. Menstruation and menstrual hygiene amongst adolescent school girls in Kano, Northwestern Nigeria. Afr J Reprod Health. 2010;14(3):201-7.

20. Das P, Baker KK, Dutta A, Swain T, Sahoo S, Das BS, et al. Menstrual Hygiene Practices, WASH Access and the Risk of Urogenital Infection in Women from Odisha, India. PLoS One. 2015;10(6):e0130777.

21. Adinma ED, Adinma JI. Menstrual characteristics amongst south-eastern Nigerian adolescent school girls. West Afr J Med. 2009;28(2):110-3.

22. Tegegne TK, Sisay MM. Menstrual hygiene management and school absenteeism among female adolescent students in Northeast Ethiopia. BMC Public Health. 2014;14:1118. 
23. Sommer M, Kjellén M, Chibesa Pensulo C. Girls' and women's unmet needs for menstrual hygiene management (MHM): the interactions between MHM and sanitation systems in low-income countries. Journal of Water, Sanitation and Hygiene for Development. 2013.

24. Garg R, Goyal S, Gupta S. India moves towards menstrual hygiene: subsidized sanitary napkins for rural adolescent girls - issues and challenges. Maternal and Child Health Journal. 2012;16:767-74.

25. Garikipati S, Boudot C. To Pad or Not to Pad: Towards Better Sanitary Care for Women in Indian Slums. J Internat Develop. 2017;29(1):32-51.

26. Crofts T, Fisher WA. Menstrual Hygiene in Ugandan Schools and investigation of low-cost sanitary pads. J Water, Sanitation Hyg Develop. 2012;2:50-8.

27. Sommer M. Ideologies of sexuality, menstruation and risk: girls' experiences of puberty and schooling in northern Tanzania. Cult Health Sex. 2009;11(4):383-98.

28. Oduor C, Alexander K, Oruko K, Nyothach E, Mason L, Odhiambo F, et al. Schoolgirls' experiences of changing and disposal of menstrual hygiene items and inferences for WASH in schools. Waterlines. 2015;34(4):397-411.

29. North B, Oldham M. Preclinical, Clinical, and Overthe-Counter Postmarketing Experience with a New Vaginal Cup: Menstrual Collection. J Womens Health. 2011;20(2).

30. Howard C, Rose CL, Trouton K, Stamm H, Marentette D, Kirkpatrick N, et al. FLOW (finding lasting options for women): multicentre randomized controlled trial comparing tampons with menstrual cups. Can Fam Physician. 2011;57(6):e208-15.

31. Stewart K, Powell M, Greer R. An alternative to conventional sanitary protection: would women use a menstrual cup? J Obstet Gynaecol. 2009;29(1):4952.

32. Stewart K, Greer R, Powell M. Women's experience of using the Mooncup. J Obstet Gynaecol. 2010;30(3):285-7.

33. Oster E, Thornton R. Menstruation, Sanitary Products and School Attendance: Evidence from a Randomized Evaluation. Am Econ J Appl Economics. 2011;3(1):91-100.

34. Nyothach E, Alexander K, Oduor C, Mason L, Oruko K, Odhiambo F, et al. Handwashing for menstrual hygiene management among primary schoolgirls in rural western Kenya. Waterlines. 2015;34(4):279-95.

35. Alexander K, Oduor C, Nyothach E, Laserson K, Amek N, Eleveld A, et al. Water, Sanitation and Hygiene Conditions in Kenyan Rural Schools: Are
Schools Meeting the Needs of Menstruating Girls? Water. 2014;6:1453-66.

36. Mason L, Laserson K, Oruko K, Nyothach E, Alexander K, Odhiambo F, et al. Adolescent schoolgirls' experiences of menstrual cups and pads in rural western Kenya: a qualitative study. Waterlines. 2015;34(1):15-30.

37. Juma J, Nyothach E, Laserson KF, Oduor C, Arita L, Ouma $\mathrm{C}$, et al. Examining the safety of menstrual cups among rural primary school girls in western Kenya: observational studies nested in a randomised controlled feasibility study. BMJ open. 2017;7(4):e015429.

38. Cohen D, Atieno-Odhiambo E. Siaya: The Historical Anthropology of an African Landscape. London: James Currey, Ltd; 1989.

39. Odhiambo FO, Laserson KF, Sewe M, Hamel MJ, Feikin DR, Adazu K, et al. Profile: The KEMRI/CDC Health and Demographic Surveillance System--Western Kenya. International journal of epidemiology. 2012;41(4):977-87.

40. Cheng M, Kung R, Hannah M, Wilansky D, Shime J. Menses cup evaluation study. Fertility And Sterility. 1995;64(3):661-3.

41. Ellis A, Haver J, Villasenor JON, Parawan A, Venkatesh M, Freeman MC, et al. WASH challenges to girls' menstrual hygiene management in Metro Manila, Masbate, and South Central Mindanao, Philippines. Waterlines. 2016;35(3):306.

42. Scorgie F, Foster J, Stadler J, Phiri T, Hoppenjans L, Rees H, et al. Bitten By Shyness: Menstrual Hygiene Management, Sanitation, and the Quest for Privacy in South Africa. Med Anthropol. 2016;35(2):161-76.

43. Adinma ED, Adinma JI. Perceptions and practices on menstruation amongst Nigerian secondary school girls. Afr J Reprod Health. 2008;12(1):74-83.

44. Everson N, Ednah B. Menstrual hygiene - A salient hazard in rural schools: A case of Masvingo district of Zimbabwe. Jàmbá. J Disaster Risk Studies. 2016;8(2):e1-e8.

45. van Eijk AM, Laserson KF, Nyothach E, Oruko K, Omoto J, Mason L, et al. Use of menstrual cups among school girls: longitudinal observations nested in a randomised controlled feasibility study in rural western Kenya. Reprod Health. 2018;15(1):139.

Cite this article as: Mason L, Nyothach E, van Eijk AM, Obor D, Alexander KT, Ngere I, et al. Comparing use and acceptability of menstrual cups and sanitary pads by schoolgirls in rural western Kenya. Int J Reprod Contracept Obstet Gynecol 2019;8:2974-82. 